\title{
Management of Pregnant Women in Times of Covid-19: A Review of Current Literature
}

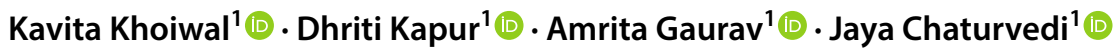

Received: 29 May 2020 / Accepted: 21 June 2020 / Published online: 7 July 2020

(c) Federation of Obstetric \& Gynecological Societies of India 2020

\begin{abstract}
Abstarct
COVID-19 is a Public Health Emergency of International Concern. Its impact on pregnant women is not yet clear owing to limited data and the knowledge is evolving in several aspects. Based on the available evidences, various clinical guidelines for management of COVID-19 have been formulated. This article intends to compile and summarise guidelines from esteemed organisations, along with their implication in the Indian scenario, and offers an easy tool for clinicians managing pregnant women in times of COVID-19.
\end{abstract}

Keywords COVID-19 $\cdot$ SARS-CoV $\cdot$ Pregnant women $\cdot$ Postpartum care

\section{Introduction}

In late December 2019, a case of unidentified pneumonia was reported in Wuhan, Hubei Province, People's Republic of China (PRC). Its clinical characteristics were very similar to those of viral pneumonia. After analysis of respiratory samples, the experts at the PRC centers for Disease Control declared that the pneumonia, later known as novel coronavirus pneumonia, was caused by a novel coronavirus [1]. WHO declared coronavirus disease 2019 (COVID-19) as a Public Health Emergency of International Concern on January 30, 2020 [2]. More than 180 countries have reported laboratory-confirmed cases of COVID-19 on all continents except Antarctica [3]. As of May 23, 2020, COVID-19 has been confirmed in over 5 million individuals worldwide and has resulted in more than 340,290 deaths [4]. In India, the numbers stand at 1,24,794 active cases and 3726 deaths and are still rising.

Coronaviruses are single-stranded RNA viruses with a diameter of $80-120 \mathrm{~nm}$. There are four types: $\alpha$-coronavirus, $\beta$-coronavirus, $\delta$-coronavirus and $\gamma$-coronavirus. Prior to SARS-CoV-2, six coronaviruses were known to cause disease in humans, including SARS-CoV and MERSCoV. SARS-CoV-2, like SARS-CoV and MERS-CoV, is a

Kavita Khoiwal

kavita.kh27@gmail.com

1 Department of Obstetrics and Gynecology, All India Institute of Medical Sciences, Rishikesh, India $\beta$-coronavirus [5]. Coronaviruses cause illness ranging in severity from the common cold to severe respiratory illness and death. Frequent manifestations include fever, cough, myalgia, headache and diarrhea. Abnormal testing includes abnormalities on chest radiographic imaging, lymphopenia, leukopenia and thrombocytopenia.

COVID-19 is a rapidly evolving situation, and there is limited data reporting its impact on pregnant women. Based on the available data, various clinical guidelines for management have been formulated. This article intends to compile and summarize guidelines from esteemed organizations, along with their implication in the Indian scenario and offers an easy tool for clinicians managing pregnant women in times of COVID-19.

\section{Overview of Articles Reviewed}

In response to the international concerns regarding the COVID-19 outbreak, The International Federation of Gynecology and Obstetrics (FIGO) issued comprehensive guidance for the management of pregnant women which was published in April, 2020 [6]. The Royal College of Obstetricians (RCOG) has also been regularly issuing practice guidelines which were last updated on May 13, 2020 [7]. Following that, The American College of Obstetricians and Gynecologists (ACOG) designed algorithms to aid clinicians in their management of pregnant women with known exposure or symptoms of COVID-19 [8]. Center for Disease 
control and Prevention (CDC) too has been updating their guidelines and advising obstetricians all over the world [9]. The FIGO guidelines offer guidance to manage women in four main settings of pregnancy that is ambulatory antenatal care in the outpatient clinics, obstetrical triage, intrapartum and postpartum management. The RCOG guidelines offer advice for health care professionals and other services caring for pregnant women. All the above guidelines intend to aid hospitals and clinicians in applying broader interim guidance on managing pregnant women in COVID times after suitable modifications as per individual scenarios in each county. Hence, this article along with reviewing the above-mentioned guidelines, compares them to the recommendations provided for India by the ICMR (Indian Council of Medical Research) [10] and FOGSI GCPR (Federation of Obstetric And Gynaecological Societies of India Good Clinical Practice Recommendation) [11], thereby offering a review for fighting the COVID-19 battle in our country.

\section{Effect of COVID-19 on Maternal and Fetal Outcome}

It has been long known that, while pregnant women are not necessarily more susceptible to viral illness, changes to their immune system during pregnancy may be associated with more severe symptoms. SARS-CoV and MERS-CoV were both known to be responsible for severe complications during pregnancy [12]. However, there is no current evidence that pregnant women are more susceptible to COVID-19 infection or those with COVID-19 are more prone to develop severe pneumonia.

RCOG guidelines emphasized on the risk of venous thromboembolism (VTE), as they suggest that COVID-19 produces a hyper-coagulable state and pregnancy itself is a hyper-coagulable state. Thus, COVID-19 during pregnancy could increase the risk of maternal VTE. Another important aspect is the existence of comorbidities in pregnancy. Presently, there is no data in this regard, yet FIGO logically assumes on the basis of evidence from non-pregnant women that the risk of acquiring and presenting with severe clinical symptoms in comorbid pregnant women would be higher. These risks apply in a similar fashion to the Indian scenario also. Additionally, ICMR and GCPR points out the increased risk of domestic violence and need for support especially in terms of mental health for women.

No vertical transmission, teratogenicity or early pregnancy loss has been reported in literature so far. In a study by Chen et al., all nine live births of COVID positive mothers had APGAR score of 8-9 and 9-10 at 1 and 5 min, respectively, and tested COVID negative [13]. RCOG points out the case reports of preterm birth in women with COVID-19 but has mentioned it to be unclear whether this was iatrogenic or spontaneous $[13,14]$. In India, COVID-19 infection is currently not an indication for medical termination of pregnancy.

\section{Management of Pregnant Women}

\section{Care in Outpatient Clinics}

All guidelines advice appointments to be taken prior to visit and screening at the entrance to hospital. FIGO further guides that screen positive patients should have minimum waiting period, evaluated for the presence of severity of symptoms and tested according to local protocols. In India, the GCPRs advice screening by using the checklist tool on telephone itself and recommends the testing of women from hotspot districts, even if asymptomatic, when presenting in labor or likely to deliver in next 5 days.

ACOG guidelines further provide an algorithm to help classify based on severity of symptoms where clinical symptoms such as difficulty in breathing/gasping/cough more than 1 teaspoon blood/chest pain other than coughing/dysphagia/confusion point toward an elevated risk. If this is not the case, then on clinical and social risk factors such as comorbidities or obstetric issues such as preterm labor, they classify her to the moderate-risk group while women with minimal symptoms and all above criteria ruled out belong to the low risk category.

FIGO suggests a detailed anomaly scan at 18-23 weeks of gestation followed by monthly ultrasound for fetal growth, and amniotic fluid for confirmed COVID-19 infected pregnant women. ICMR suggests USG for fetal growth surveillance after 14 days of resolution of acute illness. If a screen positive woman contacts the hospital before coming, the visit should be deferred by 14 days unless there is an urgent maternal or fetal indication.

Keeping in mind, the lack of feasibility of services in India and the risk of defaulters in follow-up visits, ICMR guidelines suggest that visits should be deferred until 7 days after start of symptoms and any woman who has a routine appointment delayed for more than 3 weeks, be contacted by telephone or household visits in full personal protective equipments (by the ASHA/ANM in rural areas).

In essence, the minimum in-person OPD visits suggested by FIGO are 6 in number, at 12 weeks/20 weeks/2 8 weeks $/ 32$ weeks/36 weeks and 37-41 weeks. All other visits to be by tele or videoconferencing which must be made available as a duty, by the obstetricians. RCOG adds on that on booking visit if VTE risk score is 3 or more then commencement of prophylactic Low molecular weight heparin (LMWH) should be recommended on a case-to-case basis in the light of need for self-isolation 
and otherwise suggests a similar visits' protocol. In India too, FOGSI guidelines reiterate the role of telemedicine in antenatal care and advice continued practice of ethical and professional standards as in routine care. Similar visits as suggested by FIGO should be scheduled for antenatal women in India as per ICMR and FOGSI. Specific stress must be laid on the need to come alone or only one accompaniment.

In dispensing clinical duties, we ought to not forget that the pandemic inevitably results in an increased amount of anxiety and maternal mental well-being too is our responsibility. Psychological counseling in a highly populous country like India is often passed over but needs to be stressed upon. Last, but not the least, as RCOG reiterates to counsel the women that not all usual visits are essential, but the essential visits informed are not to be ignored. The need for those appointments is greater than the risk of being exposed to COVID-19.

\section{Care in Triage Area}

In case of screen positive patients in triage for obstetrical or respiratory reasons, they should be assessed in isolated room as per the FIGO guidelines. RCOG stresses that if the woman has fever, consider COVID as a cause of fever/sepsis but do not ignore other causes and thus recommends a sepsis screen. Women with mild symptoms can be discharged after proper advice of home monitoring of symptoms while those with moderate or severe symptoms to be admitted after detailed assessment. In India, the limitations of the slum/ small household populations cannot be ignored. The service providers are thus guided by the ICMR to assess the feasibility of patient home isolation or else admit in hospital or quarantine facility. Most of these women will not require hospital admission. Still, women with tachypnea (RR $>30 /$ $\mathrm{min})$, hypoxia ( $\mathrm{SpO} 2 \leq 93 \%)$ and $>50 \%$ lung involvement on imaging must get admitted in critical care unit as per GCPR.

FOGSI recommends hydroxychloroquine $600 \mathrm{mg}$ (200 mg tds with meals) and azithromycin (500 mg OD) for 10 days for treatment of COVID-19 infection in pregnancy in addition to supportive treatment (paracetamol, oxygen). In high-risk women with uncontrolled diabetes, immunosuppression or chronic diseases, antiviral therapy (lopinavir + ritonavir or oseltamavir) may be used.

\section{Intrapartum Care}

Suspected/probable patients should be treated in isolation, and confirmed cases should be managed in a negative pressure isolation room while the critical ones should be managed in an ICU. While FIGO suggests that pregnant women with a mild clinical presentation could be initially considered for home confinement provided the monitoring be ensured, ICMR suggests that if a woman tests positive, she should be advised to deliver at least at a First Referral Unit, preferably a tertiary facility. FIGO is in consensus, suggested designated tertiary hospitals for delivery of these patients. FOGSI further recommends public and private health care in India to be organized into COVID and non-COVID facilities, and COVID facilities be designated for different levels of care.

Women with mild disease or suspected cases require general fluid-electrolyte balance and symptomatic treatment along with close vigilant maternal vitals' surveillance. Monitoring for secondary bacterial infection and timely use of antibiotics when indicated is stressed upon. In India too, obstetricians should be wary of the same and include these guidelines in their management.

Induction of labor of suspected/confirmed cases should be deferred unless strictly necessary. RCOG advices pregnant women admitted with suspected/confirmed COVID19 should receive prophylactic LMWH unless expected to deliver within next $12 \mathrm{~h}$. Thromboprophylaxis has not been stressed upon in other guidelines reviewed.

Though, the evidence is limited, yet fetal distress has been reported in the some studies [13]. Hence, all reviewed guidelines are prompt to advice continuous electronic fetal monitoring using cardiotocograph. The timing and mode of delivery should be individualized, and vaginal delivery is not contraindicated. Maternal observations, especially the oxygen saturation (maintain > 94\%), throughout labor should be dealt with utmost vigilance. Intravenous fluids to be given with caution, knowing the association of COVID-19 with acute respiratory distress syndrome [1]. Decision for shortening the second stage of labor can be considered and should be kept a low threshold for and management needs to be escalated if any signs of decompensation develop. In case of cesarean section, regional anesthesia is preferred, operation theater (OT) staff should be minimum and in PPE. FOGSI guidelines point out the difficulty in communication faced when in PPE gear and thus advice that this operating team be familiar with the standard operative steps. Non-elective/ emergency procedures should preferably be carried out in a different OT to allow time for a full postoperative theater clean.

In preterm cases requiring delivery, FIGO urges caution for the use of corticosteroids in critically ill patients as it could worsen the clinical condition. RCOG, for the lack of supportive evidence against the use, supports the use of corticosteroids when indicated.

Another point of disagreement lies in the timing of cord clamping. While FIGO vouches for an early cord clamping, RCOG still recommends delayed cord clamping provided there is no other contraindication.

All guidelines in consensus vouch for restrictions on visitors, asymptomatic birth partners, restricted to the woman's 
bedside, minimum staff working and PPE to worn near all suspected/confirmed cases.

\section{Care for Critically III Pregnant Woman}

Severe pneumonia is associated with a high maternal and perinatal mortality rate and calls for aggressive treatment. On these lines, FIGO lays down appropriate practice guidelines. Management, without doubt, has to be in a negative pressure isolation room in the ICU by a multidisciplinary team. Antenatal woman should preferably be in the left lateral position, appropriate antibiotic treatment has to be given, antiviral treatment to be promptly used, symptomatic and fluid management to continue and oxygen therapy to be made available readily, the method of ventilation being as per the patient's condition. ICMR and FOGSI recall similar guidelines.

While RCOG has been laying adequate stress on LMWH, FIGO draws attention to pathological findings in the lungs and liver of fifty COVID-19 fatalities in Italy (unpublished data) and emphasizes on further investigations into the role of LMWH in improving outcomes in severe cases before recommendations on the use of same.

As obstetricians medically indicated, preterm delivery should be considered on a case-to-case basis, and women less than 32 weeks of gestation to be transferred to a center with level 2 or level 3 neonatal ICU. The goal of all guidelines is common, to try and achieve the optimum maternal, fetal and neonatal outcome, despite the pandemic.

\section{Postpartum Care and Discharge}

There is currently insufficient evidence [15] regarding safety of breastfeeding and the need for temporary separation of the mother from baby. Limited data suggests an unlikely transmission via the breast milk, as per the CDC's latest update. Hence, the current guidelines have a common consensus, to offer expressed breast milk to neonates of critically ill mothers whereas explain the risks versus benefits of temporary separation to women with mild disease, thereby giving a choice to the mothers. In case of rooming in on the mothers' decision, hand hygiene to be done and face mask to be worn by the mothers along with a minimum distance of 2 meters or 6 feet between the mother and baby with a physical barrier in between preferably. In case of temporary separation, dedicated breast pump is to be used for expressed breast milk which has to be disinfected after each use.

RCOG advises to assess the women for risk of VTE after birth and the first dose of LMWH to be given as soon as possible if indicated, provided there has been no postpartum hemorrhage or regional anesthesia. It also advices all confirmed COVID-19 women to be prescribed at least 10 day course of LMWH on discharge.
Discharge of the neonate and mother should be planned once both of them are stable and tested negative. Majority of the postpartum visits are to be conducted telephonically and patient to be called when in person examination is unavoidable. In India, as is much needed, FOGSI recommends the widespread community awareness of recovery so as to reduce the anxiety pertaining to discharge. Another step in the same direction is to include COVID infection-related advice in addition to the usual postpartum instructions on the discharge card.

In conclusion, the literature on the management of antenatal women in COVID times is extremely limited but rapidly growing and changing. The available guidelines too are updating with time. The latest guidelines at present were reviewed, and a summary of management guidelines and their interpretation in the Indian Scenario has been attempted by this article. History of MERS or SARS threatens of the possible high mortality rates of COVID, yet the age group of the antenatal women and the current COVID antenatal data till date reassures otherwise. Appropriate and timely management seem to be the key, and all guidelines strive toward a safe motherhood and healthy offspring in the times of a global pandemic.

Funding No funding required.

\section{Compliance with Ethical Standards}

Conflicts of interest Dr. Kavita Khoiwal, Dr. Dhriti Kapur, Dr. Amrita Gaurav and Dr. Jaya Chaturvedi declare that they have no conflict of interest.

\section{References}

1. Huang C, Wang Y, Li X, et al. Clinical features of patients infected with 2019 novel coronavirus in Wuhan, China. The Lancet. 2020;395(10223):497-506.

2. Coronavirus Disease (COVID-19) —events as they happen [Internet]. https://www.who.int/emergencies/diseases/novel-coronaviru s-2019/events-as-they-happen. Accessed 23 May 2020.

3. World Health Organization. Coronavirus disease 2019 (COVID19) Situation Report-48. World Health Organization. https:// www.who.int/docs/default-source/coronaviruse/situation-repor ts/20200308-sitrep-48-covid-19.pdf?sfvrsn=16f7ccef_4. March 8, 2020; Accessed 9 Mar 2020.

4. Coronavirus Update (Live): 5,322,358 Cases and 340,290 Deaths from COVID-19 Virus Pandemic-Worldometer [Internet]. https ://www.worldometers.info/coronavirus. Accessed 23 May 2020.

5. Wang L, Wang Y, Ye D, Liu Q. Review of the 2019 novel coronavirus (SARS-CoV-2) based on current evidence. Int J Antimicrob Agents. 2020;55(6):105948.

6. Poon LC, Yang H, Kapur A, et al. Global interim guidance on coronavirus disease 2019 (COVID-19) during pregnancy and puerperium from FIGO and allied partners: Information for healthcare professionals. Int J Gynaecol Obstet. 2020;149(3):273-86. 
7. Coronavirus (COVID-19) infection and pregnancy [Internet]. Royal College of Obstetricians \& Gynaecologists. https://www. rcog.org.uk/en/guidelines-research-services/guidelines/coronaviru s-pregnancy. Accessed 26 May 2020.

8. Novel Coronavirus 2019 (COVID-19) [Internet]. https://www. acog.org/en/Clinical/Clinical-Guidance/Practice-Advisory/Artic les/2020/03/Novel-Coronavirus-2019. Accessed 26 May 2020.

9. CDC. Coronavirus Disease 2019 (COVID-19) [Internet]. Centers for Disease Control and Prevention. 2020. https://www.cdc.gov/ coronavirus/2019-ncov/hcp/inpatient-obstetric-healthcare-guida nce.html. Accessed 26 May 2020.

10. Indian Council of Medical Research. Guidance for management of pregnant women in covid-19 pandemic [internet]. India: ICMR; 2020. www.icmr.gov.in/pdf/covid/techdoc-Guidance_for_manag ement_of_pregnant_women_in_COVID19_pandemic_12042020. pdf. Accessed 26 May 2020.

11. Gandhi A, Ganatra A, Tank P (eds), FOGSI GCPR on Pregnancy with COVID-19 infection Version 2 [internet], Federation of Obstetric and Gynaecological Societies of India 2020; www.fogsi .org/fogsi-gcpr-on-pregnancy-with-covid-19-infection-version-2/. Accessed June 2020.

12. Al-Tawfiq JA. Middle East Respiratory Syndrome Coronavirus (MERS-CoV) and COVID-19 infection during pregnancy. Travel Med Infect Dis [Internet]. 2020; https://www.ncbi.nlm.nih.gov/ pmc/articles/PMC7118624. Accessed 26 May 2020.

13. Chen H, Guo J, Wang C, et al. Clinical characteristics and intrauterine vertical transmission potential of COVID-19 infection in nine pregnant women: a retrospective review of medical records. Lancet. 2020;395(10226):809-15.

14. Di Mascio D, Khalil A, Saccone G et al. Outcome of coronavirus spectrum infections (SARS, MERS, COVID-19) during pregnancy: a systematic review and meta-analysis. Am J Obstetr Gynecol MFM. 2020;2(2, Supplement):100107.

15. Zhu H, Wang L, Fang C, et al. Clinical analysis of 10 neonates born to mothers with 2019-nCoV pneumonia. Transl Pediatr. 2020;9(1):51-60.

Publisher's Note Springer Nature remains neutral with regard to jurisdictional claims in published maps and institutional affiliations.

\section{About the Author}

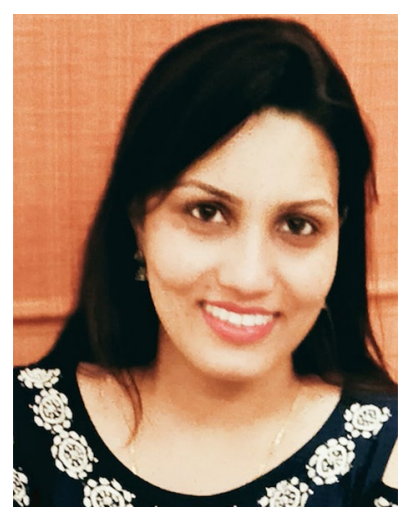

Dr. Kavita Khoiwal is working as assistant professor in the Department of Obstetrics \& Gynaecology, AIIMS Rishikesh. She has done MBBS from SMS medical college, Jaipur, and passed MD Obstetrics \& Gynaecology from All India Institute of Medical Sciences, New Delhi. She has special interest in minimally invasive surgery and high-risk obstetrics. 